\title{
Organizational and Legal Aspects of the Use of Marketing Analysis of Multivitamin Complexes for Pharmaceutical Provision of Health Diseases Among Combatants
}

\section{Andriy Gudzenko ${ }^{1}$, Valeriy Shapovalov ${ }^{2}$, Viktoriya Shapovalova ${ }^{1}$ and Valentyn Shapovalov ${ }^{1,3 *}$}

${ }^{1}$ Medical and Pharmaceutical Law, General and Clinical Pharmacy Department, Kharkiv

Medical Academy of Postgraduate Education, Kharkiv, Ukraine

${ }^{2}$ Lviv Medical Institute, Lviv, Ukraine

${ }^{3}$ Advocates Company "Apotheosis”, Kharkiv, Ukraine

*Corresponding Author: Valentyn Shapovalov, Medical and Pharmaceutical Law, General and Clinical Pharmacy Department, Kharkiv Medical Academy of

Postgraduate Education, Kharkiv, Ukraine
Received: February 18, 2021

Published: March 22, 2021

(C) All rights are reserved by Valentyn

Shapovalov., et al.

\begin{abstract}
The article presents the results of the study of the multivitamin complexes for the pharmaceutical provision of health disorders among combatants in Ukraine. In the conditions of military conflict, combatants experience various health problems and require complex pharmacotherapy and pharmaceutical provision. In that medicines of various clinical and pharmacological groups, including vitamins are widely used. The goal of the study was to conduct analysis on the organizational and legal aspects of multivitamin complexes for the pharmaceutical provision of combatants on the example of the pharmaceutical market of Ukraine in the period of 2015-2020. The organizational and legal study conducted was based on using of normative and legal, documentary, regulatory, forensic and pharmaceutical, and marketing analysis methods. Discussed the range of multivitamin complexes by trade names, composition of active substances, manufacturers, pharmaceutical forms, registration data, and parameters of the control regime. In conclusion, proved that the multivitamin complexes are highly available for pharmacotherapy and pharmaceutical provision of combatants. Keywords: Pharmaceutical Provision; Combatants; Pharmaceutical Market; Marketing Analysis; Multivitamin Complexes; Control Regime
\end{abstract}

\section{Introduction}

Military operations have a direct impact on combatants who receive traumas of different genesis that may occur during or after the war and require appropriate pharmacotherapy. Indicated in publications on the war in Iraq and Afghanistan [1,2], Columbia [3], Burundi [4], the Persian strait [5], in the east of Ukraine [6]. The formation of health diseases among combatants occurs in the zone of military conflict $[7,8]$.

The study of the experience of doctors of military hospitals shows the importance of timely pharmaceutical provision for com- batants with their subsequent long-term rehabilitation at the place of residence [9]. Studies by medical center for the study of traumatic brain injury (USA, Texas) show that combatants when using explosives [10] receive more than $50 \%$ of all injuries.

In the conditions of psycho-traumatic actions of combat stress servicemen experience anxiety (57\%), phobias (35\%), depression (48\%) and social dezaptiation (75\%) in the form of conflict, alienation and abuse of psychoactive substances (alcohol, nicotine, etc.), as a way to overcome a stressful situation against a background of various chronic (psycho-) somatic diseases [11-14]. During the 
Organizational and Legal Aspects of the Use of Marketing Analysis of Multivitamin Complexes for Pharmaceutical Provision of Health Diseases Among Combatants

complex pharmacotherapy of acquired, chronic and comorbid health disorders among the US combatants are widely used medicines of various clinical and pharmacological groups, including vitamins [5,16-18].

Vitamin pharmacotherapy helps to restore physical and mental activity among combatants, reduce the risk of colds, improve liver function, normalize memory, sleep, digestion, fat metabolism, and strengthen the immune system. According to the data obtained by Allott K., McGorry P., Yuen H., Firth J., Proffitt T., Berger G., Maruff P., O’Regan M., Papas A., Stephens T., O’Donnell C. [19] 12-week administration of $B$ vitamins has a specific neuroprotective effect on attention, alertness, especially in patients with affective psychosis.

For the pharmaceutical provision of such states at combatants, vitamins are widely used in symptomatic pharmacotherapy of mental, neurological, traumatological, narcological, cardiological, endocrinological disorders, as well as alcohol-dependent comorbidities of hepatitis, pancreatitis, tuberculosis, tobacco smoking, and others. In our previous studies we analyzed an assortment of medicines containing ethyl alcohol (ethanol) based on forensic pharmacy; assortment of medicines for pharmaceutical correction of alcoholic tremor in the structure of withdrawal syndrome of alcohol dependence; the structure of the pharmaceutical market of OTC medicines, as well as the particularities of circulation, availability and pharmaceutical provision of medicines for different contingents of the population [20-25].

Clinical and pharmacological groups of vitamins is included to the State Medicinal Products Formulary of Ukraine, clinical protocols for the provision of medical care to patients with mental and behavioral disorders due to alcohol use, cancer, diabetic neuropathy, tuberculosis, alcoholic hepatitis, etc. According to the anatomical, therapeutic and chemical classification system of the WHO (Anatomical Therapeutic Chemical (ATC) classification system, WHO, 2016), vitamins belong to the clinical and pharmacological group "A - medications, affecting the digestive system and metabolism". Pharmaceutical market of Ukraine represented by an extremely wide spectrum of vitamins in the form of multivitamin complexes for the non-prescription release. However, the uncontrolled use multivitamin complexes with high doses of vitamins can lead to overdose, the development of hypervitaminosis, hypermicroelementosis, imbalance of vitamins and even toxic effects [26].
Aim

The goal of the study was to conduct marketing analysis in the organizational and legal aspects and to determine the parameters of the control regime of multivitamin complexes according to the ATC code A11BA, which are in circulation on the pharmaceutical market of Ukraine in the period of 2016-2020 for the pharmaceutical provision of health disorders among combatants.

\section{Material and Methods}

The organizational and legal study was conducted on the example of the pharmaceutical market of Ukraine. The methodology and design of the organizational and legal research were built on the solution of eight problems: The circulation and the range of multivitamin complexes on the pharmaceutical market; Characteristics of multivitamin complexes by the composition of active substances; Distribution of manufacturers' countries of multivitamin complexes on the pharmaceutical market; Distribution of domestic manufacturers of multivitamin complexes on the pharmaceutical market; The study of the range of multivitamin complexes by medicinal forms; Distribution of multivitamin complexes by medicinal form; Distribution of multivitamin complexes by quantity of medicinal forms and manufacturers; Registration certificates validity periods of multivitamin complexes.

The organizational and legal study was conducted on basic using normative and legal, documentary, regulatory, forensic and pharmaceutical, marketing analysis methods. The normative and legal analysis was carried out based on the legal framework in force in Ukraine (laws, orders, orders, and recommendations). Regulatory and documentary analysis was based on the study of documents regulating the circulation of medicines (clinical protocols, treatment standards, instructions for medical use, medical history, prescriptions, and literature). Forensic and pharmaceutical analysis consisted in determining the clinical and pharmacological, classification and legal, nomenclature and legal groups of drugs.

The marketing analysis conducted using current legal documents, namely: the Order of the Ministry of Healthcare of Ukraine of September 21, 2009 No. 681 "On Approval of Clinical Protocols for the Provision of Medical Aid in the Specialty "Narcology"; the Order of the Ministry of Healthcare of Ukraine of September 17, 
2007 No. 554 "On approval of medical treatment protocols on the specialty "Oncology"; the Order of the Ministry of Healthcare of Ukraine dated May 22, 2009 No. 356 "On approval of medical treatment protocols on the specialty "Endocrinology"; the Order of the Ministry of Healthcare of Ukraine dated September 04, 2014 No. 620 "Unified clinical protocol of primary, secondary (specialized) and tertiary (highly specialized) medical care to adults. Tuberculosis"'; the Order of the Ministry of Healthcare of Ukraine dated November 6, 2014, No. 826 "Unified clinical protocol of primary, secondary (specialized) medical aid. Alcoholic Hepatitis"; the Order of the Ministry of Healthcare of Ukraine of April 03, 2017 No. 363 "On approval of the ninth issue of the State Formulary of Medicines and ensuring its availability", etc. The definition of control regime of multivitamin complexes carried out in accordance with the current legislation of Ukraine according to the method [27,28].

\section{Results and Discussion}

Organizational and legal researches included the marketing, comparative, forensic and pharmaceutical analysis of multivitamin complexes according to the ATC code A11BA, conducted in the following areas: the study of the range of multivitamin complexes with trade names; the characteristic of multivitamin complexes in the composition of active substances; distribution of multivitamin complexes by manufacturers on the pharmaceutical market; analysis of the range of multivitamin complexes in pharmaceutical forms; a comparative analysis of multivitamin complexes by the number of dosage forms and manufacturers; studying of the terms of validity of the registration certificates of multivitamin complexes; determination of parameters of the control regime for multivitamin complexes.

Based on the conducted researches it was determined that multivitamin complexes according to the ATC belong to the subgroup A11BA - Multivitamin complexes without additives. The range of multivitamin complexes (A11VA), which are in circulation on the pharmaceutical market of Ukraine, given in the table 1.

Discussion of the research results of the circulation and the range of multivitamin complexes on the pharmaceutical market of Ukraine (Table 1) shows that they contains up 16 active substance. The multivitamin complexes are in one clinical and pharmacological group: Multivitamin complexes without additions. The multivitamin complexes have the same ATC code - A11BA.

Characteristics of multivitamin complexes by the composition of active substances presented in table 2 .

Discussion of the results after researches of the characteristics of multivitamin complexes by the composition of active substances

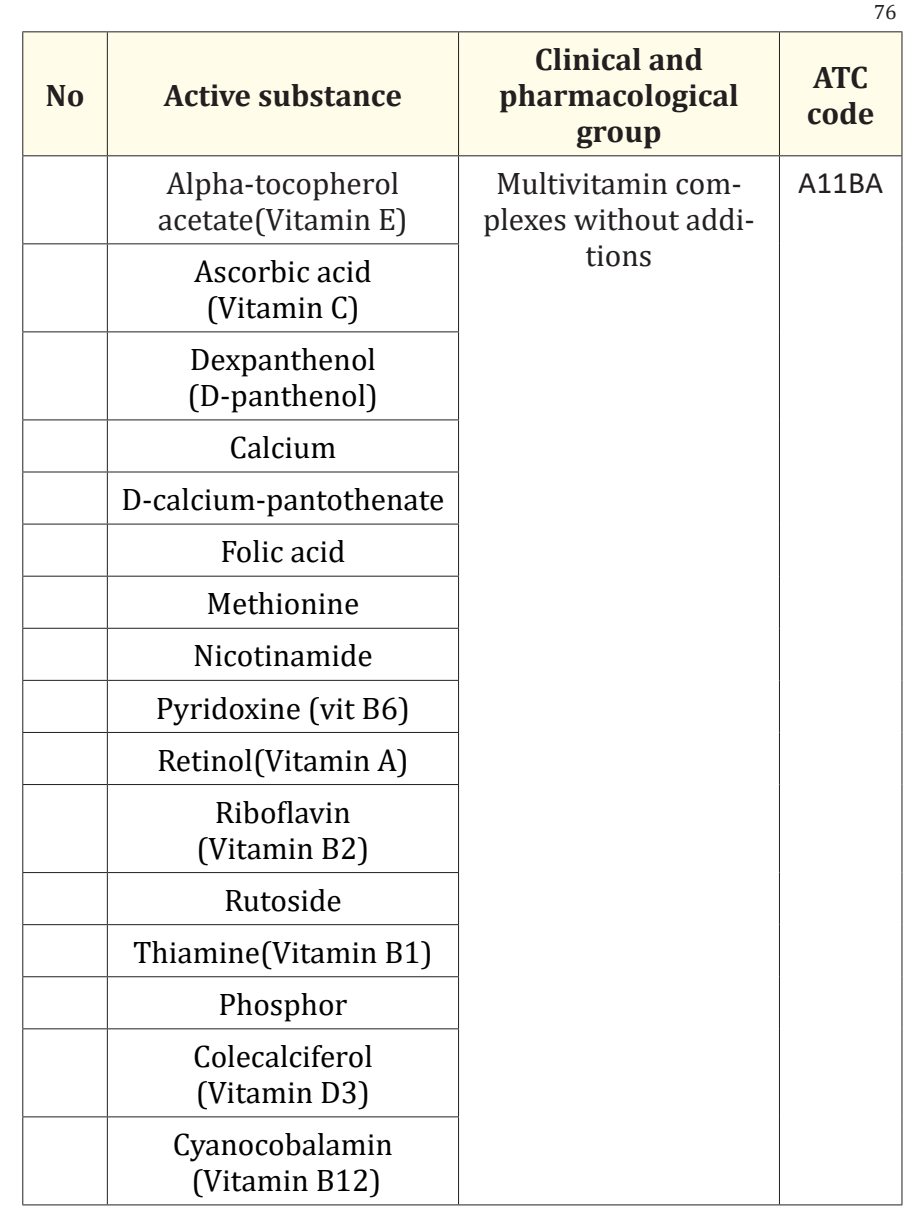

Table 1: The range of multivitamin complexes in circulation on the pharmaceutical market of Ukraine.

(Table 2) showed that the composition of all multivitamin complexes consist from Ascorbic acid and Riboflavin in various amounts. However, the composition of some multivitamin complexes differs from other available active ingredients: Dexpanthenol (Pikovit), Methionine (Decamevit), Calcium and Phosphor (Pikovit, Pikovit D).

Discussion of the results of the analysis of the distribution of manufacturers' countries of multivitamin complexes on the pharmaceutical market manufacturers showed that on the pharmaceutical market of Ukraine four multivitamin complexes are foreign (Pikovit, Syrup, Pikovit, Pikovit D, Pikovit forte) and twelve multivitamin complexes produced in Ukraine (in particular, Hexavit, Decamevit, Complevit, Revit, Undevit, Undetab), which is $75 \%$. Regarding the foreign multivitamin complexes, found that they shipped to Ukraine from Slovenia; their number in this segment of the market is $25 \%$ (Figure 1).

Discussion of the results of the distribution of domestic manufacturers of multivitamin complexes on the pharmaceutical market showed that established three pharmaceutical manufacturers 
Organizational and Legal Aspects of the Use of Marketing Analysis of Multivitamin Complexes for Pharmaceutical Provision of Health Diseases Among Combatants

\begin{tabular}{|c|c|c|c|c|c|c|c|c|}
\hline \multirow{3}{*}{$\begin{array}{c}\text { Active } \\
\text { substance }\end{array}$} & \multicolumn{8}{|c|}{ Trade names } \\
\hline & Hexavit & Decamevit & Complevit & Pikovit & Pikovit, Pikovit D & Pikovit forte & Revit & Undevit, Undetab \\
\hline & \multicolumn{8}{|c|}{ Quantity of active substances in 1 dragee, pill, capsule, $5 \mathrm{ml}$ of syrup } \\
\hline $\begin{array}{l}\text { Alpha-tocopherol } \\
\text { acetate(Vitamin E) }\end{array}$ & - & $10 \mathrm{mg}$ & - & - & - & $15 \mathrm{mg}$ & - & $10 \mathrm{mg}$ \\
\hline Ascorbic acid (Vitamin C) & $70 \mathrm{mg}$ & $200 \mathrm{mg}$ & $100 \mathrm{mg}$ & $50 \mathrm{mg}$ & $10 \mathrm{mg}$ & $60 \mathrm{mg}$ & $35 \mathrm{mg}$ & $75 \mathrm{mg}$ \\
\hline Dexpanthenol (D-panthenol) & - & - & - & $2 \mathrm{mg}$ & - & - & - & - \\
\hline Calcium & - & - & - & - & $12,5 \mathrm{mg}$ & - & - & - \\
\hline D-calcium-pantothenate & - & - & $25 \mathrm{mg}$ & - & $1,2 \mathrm{mg}$ & $10 \mathrm{mg}$ & - & $3 \mathrm{mg}$ \\
\hline Folic acid & - & $2 \mathrm{mg}$ & $0,25 \mathrm{mg}$ & - & $0,04 \mathrm{mg}$ & $0,40 \mathrm{mg}$ & - & $0,07 \mathrm{mg}$ \\
\hline Methionine & - & $200 \mathrm{mg}$ & - & - & - & - & - & - \\
\hline Nicotinamide & $15 \mathrm{mg}$ & $50 \mathrm{mg}$ & $50 \mathrm{mg}$ & $5 \mathrm{mg}$ & $3,0 \mathrm{mg}$ & $20 \mathrm{mg}$ & - & $20 \mathrm{mg}$ \\
\hline Pyridoxine (vit B6) & $2 \mathrm{mg}$ & $20 \mathrm{mg}$ & $10 \mathrm{mg}$ & $0,6 \mathrm{mg}$ & $0,3 \mathrm{mg}$ & $2 \mathrm{mg}$ & - & $3 \mathrm{mg}$ \\
\hline Retinol (Vitamin A) & $\begin{array}{c}1,72 \mathrm{mg} \\
\text { or } 2,75 \\
\mathrm{mg}\end{array}$ & $6600 \mathrm{IU}$ & - & $900 \mathrm{IU}$ & $600 \mathrm{IU}$ & $5000 \mathrm{IU}$ & $\begin{array}{c}0,86 \mathrm{mg} \\
\text { or } 1,38 \\
\mathrm{mg}\end{array}$ & $1,135 \mathrm{mg}$ \\
\hline Riboflavin (Vitamin B2) & $2 \mathrm{mg}$ & $10 \mathrm{mg}$ & $15 \mathrm{mg}$ & $1 \mathrm{mg}$ & $0,3 \mathrm{mg}$ & $1,70 \mathrm{mg}$ & $1 \mathrm{mg}$ & $2 \mathrm{mg}$ \\
\hline Rutoside & - & $20 \mathrm{mg}$ & - & - & - & - & - & $10 \mathrm{mg}$ \\
\hline Thiamine (Vitamin B1) & $2 \mathrm{mg}$ & $20 \mathrm{mg}$ & $15 \mathrm{mg}$ & $1 \mathrm{mg}$ & $0,25 \mathrm{mg}$ & $1,50 \mathrm{mg}$ & $1 \mathrm{mg}$ & $2 \mathrm{mg}$ \\
\hline Phosphor & - & - & - & - & $10,0 \mathrm{mg}$ & - & - & - \\
\hline $\begin{array}{l}\text { Colecalciferol } \\
\text { (Vitamin D3) }\end{array}$ & - & - & - & $100 \mathrm{IU}$ & $80 \mathrm{IU}$ & $400 \mathrm{IU}$ & - & - \\
\hline $\begin{array}{l}\text { Cyanocobalamin } \\
\text { (Vitamin B12) }\end{array}$ & - & $0,1 \mathrm{mg}$ & $0,002 \mathrm{mg}$ & $1 \mathrm{mcg}$ & $0,2 \mathrm{mcg}$ & $6 \mathrm{mcg}$ & - & $0,002 \mathrm{mg}$ \\
\hline
\end{tabular}

Table 2: Characteristics of multivitamin complexes by the composition of active substances.

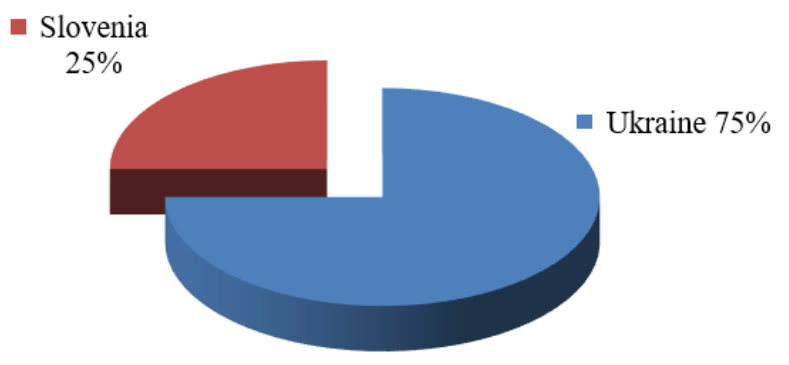

Figure 1: Distribution of manufacturers' countries of multivitamin complexes on the pharmaceutical market of Ukraine.

provide the assortment of domestic multivitamin complexes: PJSC “Vitamins", PJSC “Kyiv Vitamin Plant”, PJSC “Technolog” (Figure 2). Among the national producers, the majority of multivitamin complexes (50\%) is produced by PJSC "Kiev Vitamin Plant" (Hexavit, Decamevit, Complevit, Revit, Undevit, Undetab).

The next stage of the research was the study of the range of multivitamin complexes by medicinal forms (Table 3 ).

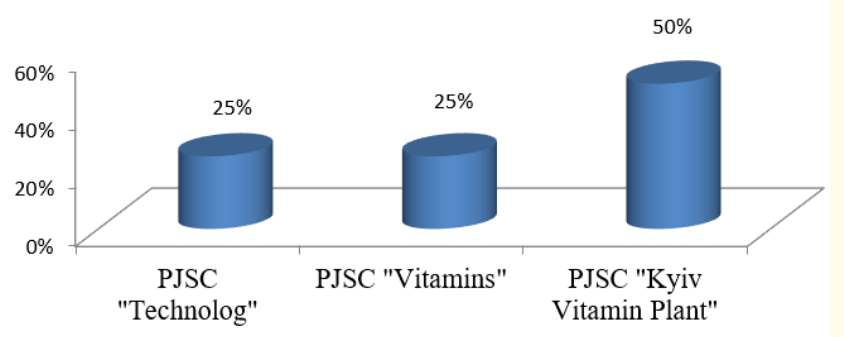

Figure 2: Distribution of domestic manufacturers of multivitamin complexes on the pharmaceutical market of Ukraine.

Discussion of the study of the range of multivitamin complexes by medicinal forms shows the following results. According to the results of the study (Table 3, Figure 3), established that multivitamin complexes represented in the majority on the pharmaceutical market of Ukraine in the form of dragee (56.25\%) and pills $(31.25 \%)$, and in the minority - in the form of syrup and capsules (6.25\% equally). 


\begin{tabular}{|c|c|c|c|}
\hline No & INN & Trade name & Medicinal form \\
\hline 1 & \multirow[t]{16}{*}{ Comb drug } & \multirow[t]{2}{*}{ Pikovit } & Syrup \\
\hline 2 & & & Coated pills \\
\hline 3 & & Pikovit D & Coated pills \\
\hline 4 & & Pikovit forte & Coated pills \\
\hline 5 & & Decamevit & Coated pills \\
\hline 6 & & Complevit & Capsules \\
\hline 7 & & \multirow[t]{3}{*}{ Undevit } & Dragee \\
\hline 8 & & & Dragee \\
\hline 9 & & & Dragee \\
\hline 10 & & \multirow[t]{3}{*}{ Revit } & Dragee \\
\hline 11 & & & Dragee \\
\hline 12 & & & Dragee \\
\hline 13 & & \multirow[t]{3}{*}{ Hexavit } & Dragee \\
\hline 14 & & & Dragee \\
\hline 15 & & & Dragee \\
\hline 16 & & Undetab & Coated pills \\
\hline
\end{tabular}

Table 3: The range of multivitamin complexes by medicinal forms.

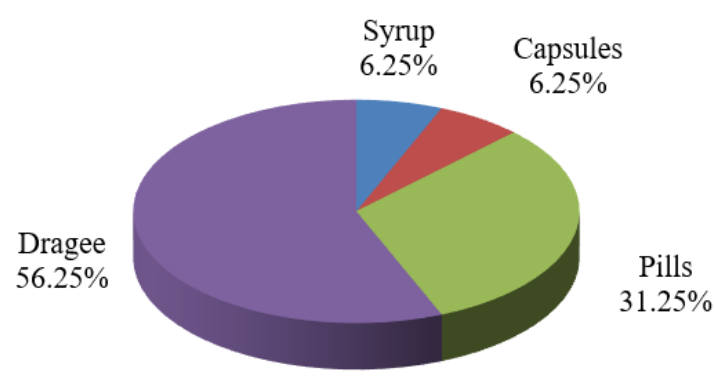

Figure 3: Distribution of multivitamin complexes by medicinal form.

The comparative characteristic of multivitamin complexes by the number of dosage forms and number of manufacturers pre sented on figure 4 .

Discussion of the results of the study about the distribution of multivitamin complexes by quantity of medicinal forms and manufacturers showed that Pikovit presented in two pharmaceutica forms - in the form of syrup and pills, and all other multivitamin complexes - in one form. At the same time, Hexavit, Revit and Undevit multivitamin complexes manufactured by three domestic pharmaceutical companies.

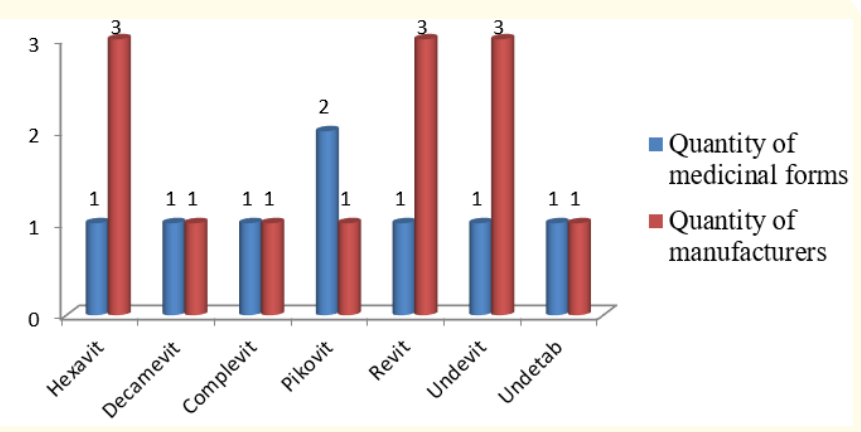

Figure 4: Distribution of multivitamin complexes by quantity of medicinal forms and manufacturers

During the study if the registration data of multivitamin complexes (Figure 5), established that five of them have unlimited registration certificates on the territory of Ukraine - Pikovit (pills),
Hexavit, Undevit, Pikovit forte, Pikovit D; one of them (Undevit) has the validity period of the registration certificate of 2013-2018; another one (Complevit) - 2014-2019; three of them (Undevit, Hexavit, Revit) - 2015-2020; six of them (Revit, Pikovit (syrup), Revit, Hexavit, Undetab, Decamevit) - 2016-2021.

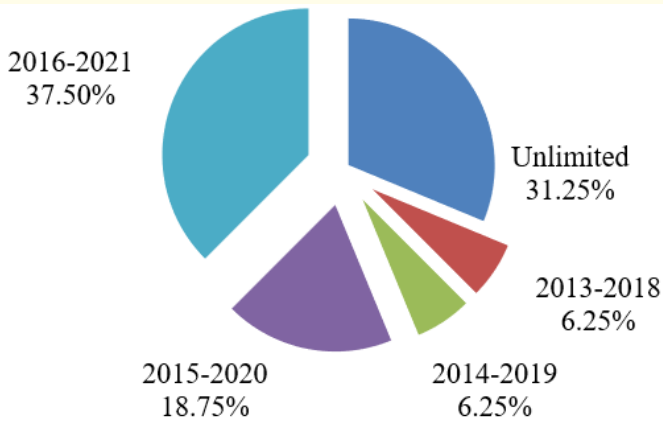

Figure 5: Registration certificates validity periods of multivitamin complexes.

Based on the above and in accordance with the current pharmaceutical legislation of Ukraine, the parameters of the control regime of multivitamin complexes that are in circulation on the pharmaceutical market of Ukraine can be represented schematically: Control regime by code A11BA + General classification and legal group + Non-prescription (OTC) nomenclature and legal group.

Discussion of the results obtained during the study showed the following main aspects. Firstly, all multivitamin complexes contain Ascorbic acid and Riboflavin in different amounts. Among of the multivitamin complexes the Ukrainian manufacturers represent $75 \%$. Only Slovenia supplies multivitamin complexes to Ukraine.

Then, the most significant of the multivitamin complexes presented in the form of pills (56.25\%) and tablets (31.25\%). Pikovit has the largest honeycomb in the form of two dosage forms (syrup and tablets). Three domestic pharmaceutical producers make multivitamin complexes Hexavit, Revit and Undevit. During studying the registration data of the multivitamin complexes, established that five multivitamin complexes have an unlimited registration period on the territory of Ukraine - Pikovit (tablets), Hexavit, Undevit, Pikovit forte, Pikovit D.

Lastly, determined the parameters of the control regime of multivitamin complexes according to the current legislation of Ukraine. Multivitamin complexes belong to the code A11BA of the ATC system according to the clinical and pharmacological group. They are included to the general group according to the classification and legal group. They released without a doctor's prescription according to the nomenclature and legal group.

These results support the conclusion that the clinical and pharmacological group of vitamins is available for pharmacotherapy and pharmaceutical support of combatants.

These results support the conclusion that the control regime of the multivitamin complexes is available for pharmacotherapy and pharmaceutical provision of combatants.

\section{Conclusion}

Within the organizational and legal aspects of pharmaceutical provision of health disorders among combatants, carried out a marketing analysis of multivitamin complexes of ATC code A11BA. 
Organizational and Legal Aspects of the Use of Marketing Analysis of Multivitamin Complexes for Pharmaceutical Provision of Health Diseases Among Combatants

Determined the assortment of multivitamin complexes according to trade names, composition of active substances, manufacturer, dosage forms, registration data, and parameters of control regime. Established that in circulation on the pharmaceutical market of Ukraine there are 16 trade names of multivitamin complexes of ATC code A11BA. When studying the composition of the multivitamin complexes, found that Ascorbic acid (vitamin C) and Riboflavin (vitamin B2) in different quantities are contained in all of the studied multivitamin complexes. However, unlike other multivitamin complexes, Pikovit contains Dexpanthenol, Decamevit - Methionine, Pikovit and Pikovit D - Calcium and Phosphor. The analysis of manufactures of multivitamin complexes showed that $75 \%$ of them produced by domestic manufacturers and $25 \%$ by foreign (KRKA, Novo mesto, Slovenia). The product range of national production provided by three pharmaceutical manufacturers, among which the majority of multivitamin complexes (50\%) produced by PJSC "Kiev Vitamin Plant" (Hexavit, Decamevit, Complevit, Revit, Undevit, Undetab). Revealed that the majority of multivitamin complexes represented on the pharmaceutical market of Ukraine in the form of dragees (56.25\%) and pills (31.25\%). In the course of studying the registration data of multivitamin complexes, established that five of them have an unlimited registration period on the territory of Ukraine, while six of them expire in various periods 2016-2021. The results of determining the parameters of the multivitamin complexes control regime showed that the clinical and pharmacological group of multivitamin complexes refer to ATC code A11VA; classification and legal group - general; nomenclature and legal group - non-prescription (OTC). Proved that the multivitamin complexes are highly available for pharmacotherapy and pharmaceutical provision of combatants.

\section{Bibliography}

1. K Seal., et al. "Bringing the war back home: mental health disorders among 103,788 US veterans returning from Iraq and Afghanistan seen at Department of Veterans Affairs facilities". Archives of Internal Medicine 167.5 (2007): 476-482.

2. R H Pietrzak., et al. "Posttraumatic stress disorder mediates the relationship between mild traumatic brain injury and health and psychosocial functioning in veterans of Operations Enduring Freedom and Iraqi Freedom". The Journal of Nervous and Mental Disease 197.10 (2009): 748-753.
3. Baez S., et al. "Ex-Combatants' Minds: Toward Situated Reintegration Process in Post-conflict Colombia”. Frontiers in Psychology 10 (2019): 73.

4. Meyer-Parlapanis D., et al. "Appetitive Aggression in Women: Comparing Male and Female War Combatants". Frontiers in Psychology 6 (2015): 1972.

5. $\quad$ R B McFee. "Gulf War Servicemen and Servicewomen: The Long Road Home and the Role of Health Care Professionals to Enhance the Troops' Health and Healing". Disease-a-Month 54.5 (2008): 265-333.

6. AO Gudzenko., et al. "Organization of pharmaceutical provision for military personnel engaged in combat operations based on pharmaceutical law". ScienceRise: Pharmaceutical Science 3.3 (2016): 20-28.

7. US department of the army. "Combat and operational stress control manual for leaders and soldiers". Washington, DC: DA. Field Manual 6 - 22.5 (2009).

8. McCarroll J E., et al. "Deployment and the probability of spousal aggression by US Army soldiers". Military Medicine 165 (2000): 41-44.

9. D. Aedo-Martín., et al. "Use of tranexamic acid in combant casualties. Experience of the Spanish medical corps. Clinical series and literature review". Revista Española de Cirugía Ortopédica y Traumatología (English Edition) 60.3 (2016): 200-205.

10. DS DeWitt and D S Prough. "Blast-Induced Brain Injury and Posttraumatic Hypotension and Hypoxemia". Journal of Neurotrauma 26.6 (2009): 877-887.

11. V Shapovalov., et al. "Organizational and legal analysis of the pharmaceutical provision for the most common diseases of society". International Journal of Pharmaceutical Sciences Review and Research 51.1 (2018): 118-124.

12. US department of the army. "Combat and operational stress control manual for leaders and soldiers". Washington, DC: DA (2009). 
Organizational and Legal Aspects of the Use of Marketing Analysis of Multivitamin Complexes for Pharmaceutical Provision of Health Diseases Among Combatants

13. DW King., et al. "Posttraumatic stress disorder in a national sample of female and male Vietnam veterans: risk factors, war-zone stressors, and resilience-recovery variables". Journal of Abnormal Psychology 108 (1999): 164-170.

14. J E McCarroll., et al. "Deployment and the probability of spousal aggression by US Army soldiers". Military Medicine 165 (2000): 41-44.

15. J Kulak., et al. "Substance use and mental health among military spouses and partners". Military Behavioral Health 7.3 (2019): 257-267.

16. J Devonish., et al. "The impact of military service and traumatic brain injury on the substance use norms of Army Reserve and National Guard Soldiers and their spouses". Addictive Behaviors 72 (2017): 51-56.

17. Arieh Y Shalev. "Posttraumatic Stress Disorder (PTSD) and Stress Related Disorders". Psychiatric Clinics of North America 32.3 (2009): 687-704.

18. M Larson., et al. "Military Combat Deployments and Substance Use: Review and Future Directions". Journal of Social Work Practice in the Addictions 12.1 (2012): 6-27.

19. K Allott., et al. "The Vitamins in Psychosis Study: A Randomized, Double-Blind, Placebo-Controlled Trial of the Effects of Vitamins B12, B6, and Folic Acid on Symptoms and Neurocognition in First-Episode Psychosis". Biological Psychiatry 86.1 (2019): 35-44.

20. Shapovalov (jr.) ., et al. "Organizational, legal and marketing characteristics of domestic medicines for pharmaceutical provision to victims suffered from emergency situations in Ukraine". The Pharma Innovation Journal 7.6 (2018): 586-589.

21. V V Shapovalov., et al. "Clinical and pharmacological, classification and legal, nomenclature and legal distribution of medicines for pharmacotherapy of depression among combatants (F 32-33)". The Health of Society 4 (2018): 181-186.
22. G V Bondarchuk., et al. "Organization and legal approaches to the circulation of medicines in provision of medical and pharmaceutical assistance to victims with traumatic injures bases on pharmaceutical law". Pharmacom 3 (2016): 129-138.

23. V Shapovalov (Jr)., et al. "Concerning the importance of forensic and pharmaceutical researches to improve patients' accessibility to medicines". Pharmacia 65.2 (2017): 23-29.

24. V Shapovalov (Jr)., et al. "Forensic and pharmaceutical study of the presence of a causal link between the degree of alcohol abuse and qualification level of the respondents". Pharmacia 64.3 (2017): 31-39.

25. S Shunkina and B Hromovyk. "Methadone as an alternative analgesic for palliative patients". Pharmacia 63.4 (2016): 21-28.

26. ATC-classification. Compendium on-line.

27. ABC and VED analysis in medical stores inventory control.

28. State formulary of medicines (Issue 9), approved by the order of the Ministry of healthcare of Ukraine dated April 3 (2017): 363.

\section{Assets from publication with us}

- Prompt Acknowledgement after receiving the article

- Thorough Double blinded peer review

- Rapid Publication

- Issue of Publication Certificate

- High visibility of your Published work

Website: https://www.actascientific.com/

Submit Article: https://www.actascientific.com/submission.php

Email us: editor@actascientific.com

Contact us: +919182824667 